\title{
Difficult to Diagnose the Cause of Intestinal Obstruction due to Abdominal Cocoon Syndrome
}

\author{
Cemal Ulusoy $^{1}$, Andrej Nikolovski $^{2}$, Nadi Nazım Öztürk $^{1}$ \\ ${ }^{1}$ Prof. Dr. Cemil Taşçıoğlu Şehir Hastanesi, Istanbul, Turkey \\ 2University Surgery Clinic "Sv. Naum Ohridski", Skopje, North Macedonia
}

Received: 24/04/2021

Accepted: $28 / 04 / 2021$

Published: $12 / 05 / 2021$

How to cite this article: Ulusoy C, Nikolovski A, Öztürk NN. Difficult to diagnose the cause of intestinal obstruction due to abdominal cocoon syndrome. EJCRIM 2021;8: doi:10.12890/2021_002588.

Conflicts of Interests: The authors declare there are no competing interests.

This article is licensed under a Commons Attribution Non-Commercial 4.0 License

\section{ABSTRACT}

Abdominal cocoon syndrome (sclerosing encapsulating peritonitis) is a rare condition associated with clinical signs of intestinal dysfunction, episodes of small bowel obstruction and sometimes a palpable abdominal mass. We present the case of a 46-year-old male patient with clinical signs of intestinal obstruction caused by primary sclerosing encapsulating peritonitis.

\section{LEARNING POINTS}

- Abdominal cocoon syndrome is rare condition with repeated episodes of intestinal obstruction.

- Radiological investigations combined with a high index of suspicion are mandatory for establishing the diagnosis.

- Proper and timely diagnosis can lead to successful conservative treatment.

\section{KEYWORDS}

Abdominal cocoon syndrome, sclerosing encapsulating peritonitis, intestinal obstruction

\section{INTRODUCTION}

Abdominal cocoon syndrome, also known as sclerosing encapsulating peritonitis (SEP), is a condition in which the small intestine is partially or completely encapsulated by a fibrocollagenous membrane. It is difficult to diagnose preoperatively ${ }^{[1]}$. We present the case of a $46-y e a r-$ old patient with small bowel obstruction symptoms diagnosed intraoperatively and successfully treated with surgery.

\section{CASE DESCRIPTION}

A 46-year-old male patient was admitted to our gastrointestinal surgery clinic with abdominal pain, nausea, vomiting and a mass in the abdomen which he had discovered himself. His medical history was otherwise unremarkable. However, two to three times in the past he had experienced abdominal pain, nausea and vomiting attacks that spontaneously regressed within 1-2 days after onset. The physical examination revealed abdominal distension, auscultatory hypoactive intestinal movements and a palpable mass in the upper left quadrant of the abdomen. Blood tests showed no abnormalities. Abdominal computed tomography (CT) showed dilatation of the stomach and the first, second and third parts of the duodenum, and a capsule encircling the intestinal loops (Fig. 1). Proximal jejunal loops were dilated with a diffusely thickened wall and post-dilation stenosis. Upper gastrointestinal endoscopy and colonoscopy findings were normal. Abdominal computed angiography was performed and showed all mesenteric vessels were patent with no signs of compression, filling defects or stenosis. Magnetic resonance (MR) enterography showed delayed gastric emptying, duodenal dilatation and stenosis and wall thickening of the proximal jejunal segments (Fig. 2). 


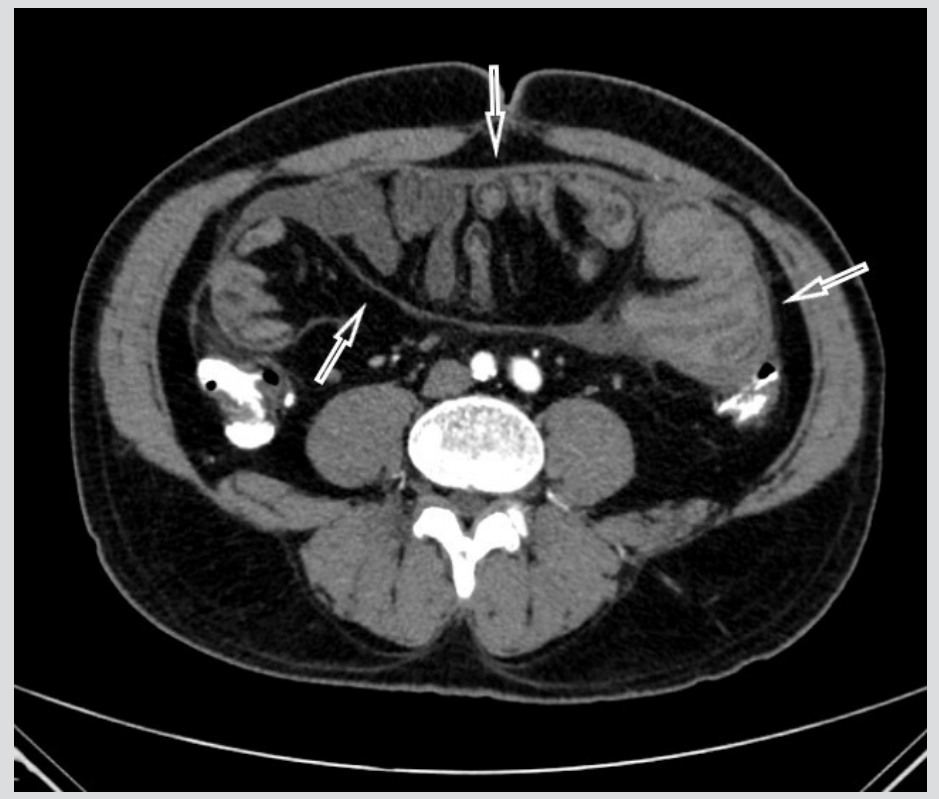

Figure 1. Abdominal computed tomography showing intestinal loops encircled with a capsule (arrows)

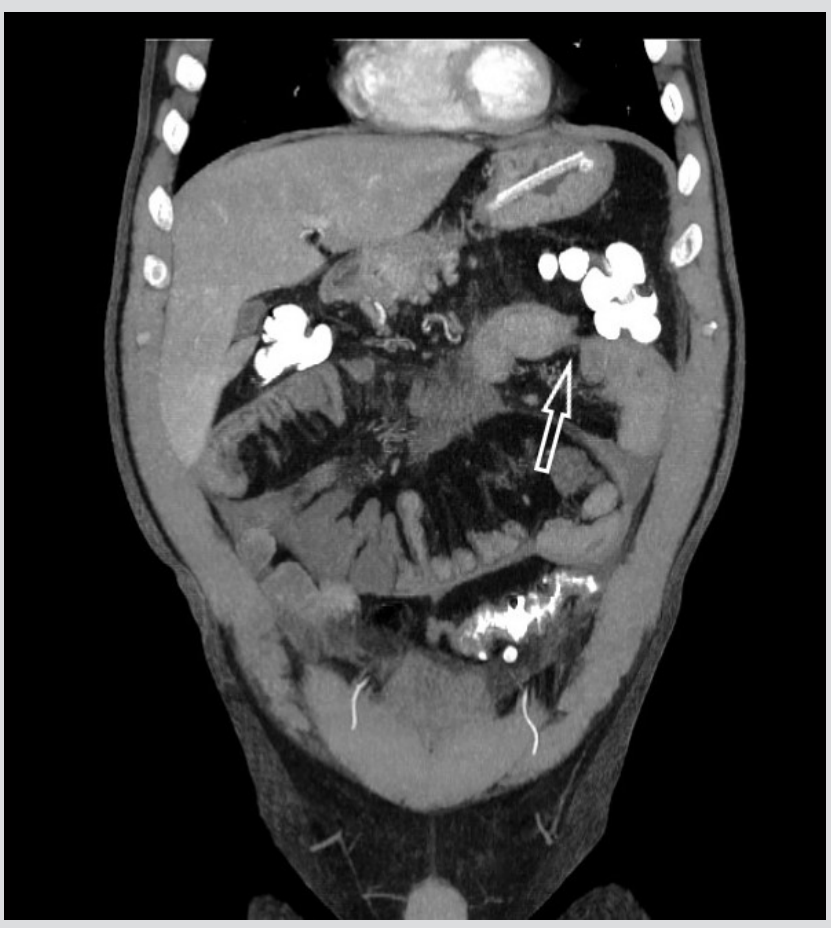

Figure 2. Magnetic resonance enterography showing wall thickening of the proximal jejunal segments with stenosis (arrow)

Oral intake was interrupted and total parenteral nutrition introduced. A nasogastric tube was placed for decompression. After electrolyte correction and fluid replacement, exploratory laparotomy was performed. Intraoperatively, the liver and the spleen were seen to have adhered to the diaphragm. The small bowel was encapsulated with a thick fibrous membrane all the way from the ligament of Treitz to the terminal ileum (Fig. 3). Bowel movements were restricted and the length of the small intestine was shorter than usual. The bowel was freed with adhesiolysis and the membranous sac removed. The postoperative period was uneventful and the patient was discharged on postoperative day 6. At 3-month follow-up, physical examination and abdominal x-ray showed no signs of intestinal obstruction.

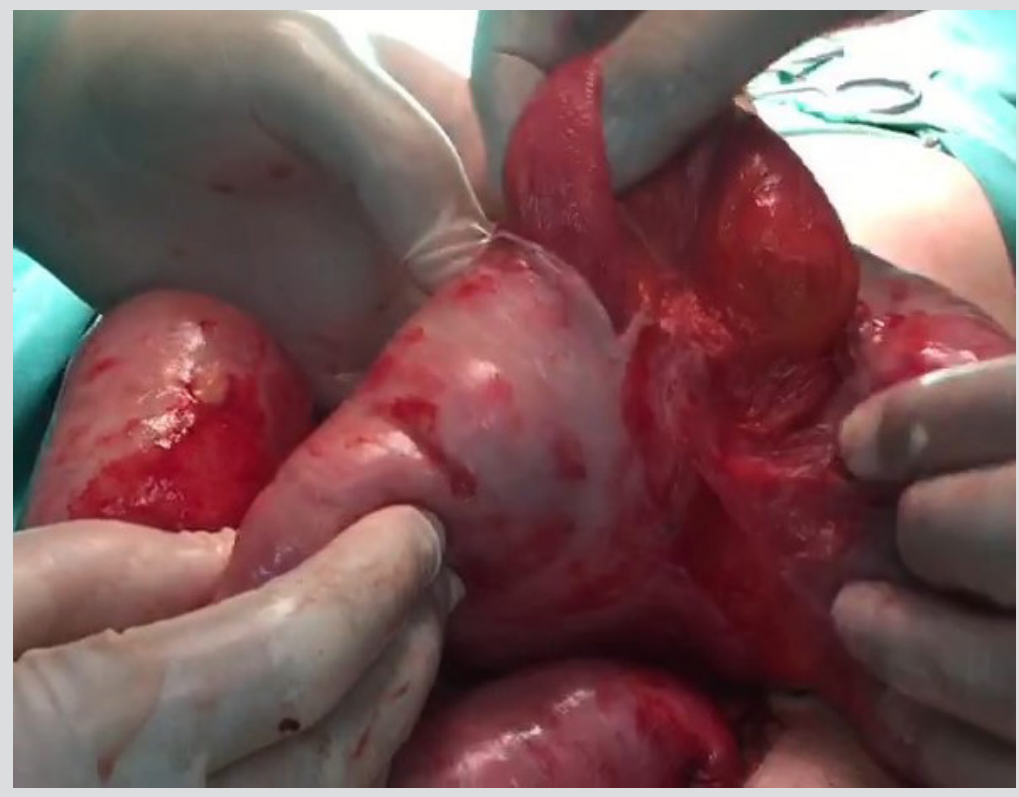

Figure 3. Small bowel encapsulated with a thick fibrous membrane 
The pathologist reported mononuclear inflammatory cells, multinuclear giant cells and degenerated mesothelial cells in the peritoneal fluid. Fibrous tissue fragments, hyalinized lipomatosis tissue and multinuclear inflammatory cells were reported in the tissue specimens.

\section{DISCUSSION}

Sclerosing encapsulating peritonitis is a condition in which the small intestine is wrapped with a thick fibrocollagenous membrane. It may be the cause of acute or chronic obstruction ${ }^{[1]}$ and is more common in females. The aetiology is unclear and it can be primary (idiopathic) or secondary. Primary SEP is also called abdominal cocoon syndrome. Continuous ambulatory peritoneal dialysis (CAPD), the presence of a LeVeen shunt, abdominal surgery, retrograde menstruation, systemic lupus erythematosus, sarcoidosis, familial Mediterranean fever and beta-blockers can cause secondary SEP ${ }^{[2]}$.

SEP is often an asymptomatic condition and may be diagnosed incidentally during abdominal surgery for other conditions. When symptomatic, it usually presents with acute or chronic symptoms of intestinal obstruction (partial or complete) accompanied by abdominal pain, nausea, vomiting and abdominal distension ${ }^{[3,4]}$. As these signs are not specific for abdominal cocoon, the preoperative diagnosis can be difficult. The reported accuracy of preoperative diagnostics for this condition is $16.7-48.7 \%{ }^{[5]}$. A high index of suspicion is mandatory for preoperative diagnosis.

In our case, the differential diagnosis included superior mesenteric artery syndrome and Crohn's disease. Both were excluded with upper gastrointestinal endoscopy, mesenteric artery computerized tomography angiography and magnetic resonance enterography. However, these examinations did not indicate abdominal cocoon. CT is the most important method for diagnosing abdominal cocoon syndrome. In this case, CT showed dilatation of the stomach and jejunum, and wall thickening of intestinal loops covered with a peritoneal sac. After repeat postoperative analysis of the CT scan, we found the 'cauliflower sign' (Fig. 4) which suggested abdominal cocoon ${ }^{[6,7]}$. MR enterography signs are similar to $\mathrm{CT}$ signs. If the CT scan indicates an abdominal cocoon, MR enterography is unnecessary for these patients.

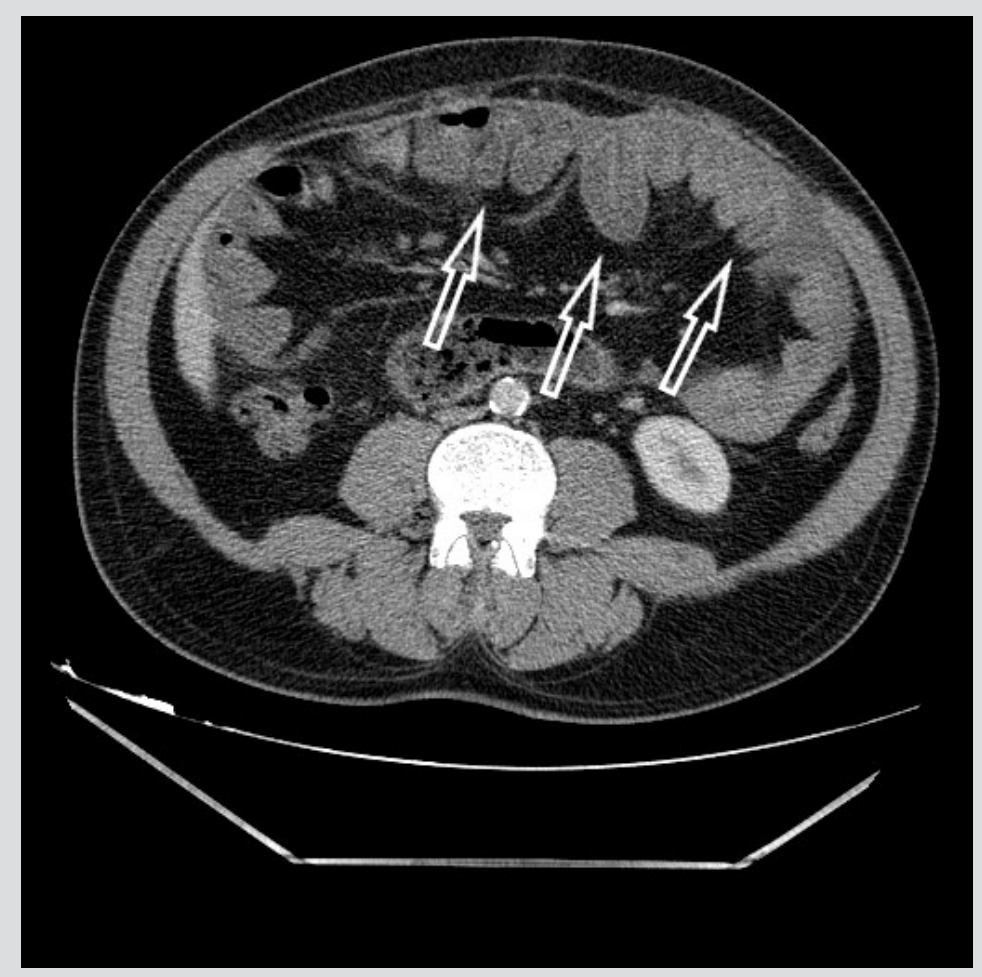

Figure 4. CT scan showing the "cauliflower sign" (arrows)

The treatment of SEP depends on whether it is diagnosed preoperatively or during surgical exploration. Patients with mild to moderate disease can be treated successfully with bowel rest, intestinal decompression and adequate nutrition (enteral and parenteral) ${ }^{[8]}$. When recurrent attacks lead to weigh loss and malnutrition or the condition presents with severe intestinal obstruction, surgical management is required. Various surgical options are available including partial or total membrane excision, adhesiolysis, resection and peeling or stripping the membrane from the intestinal serosa ${ }^{[5,9]}$. 


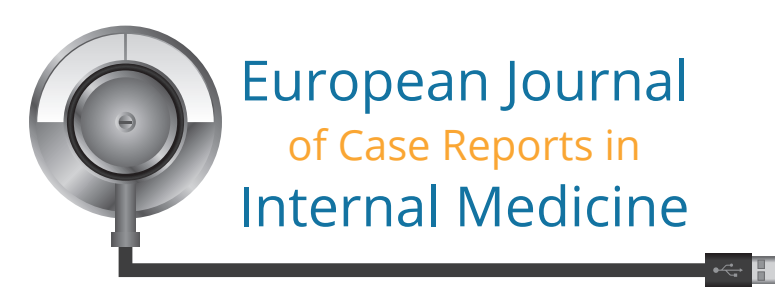

The preoperative diagnosis of abdominal cocoon syndrome is still difficult for clinicians despite current diagnostic tools and it is usually possible during laparotomy. CT findings should be examined carefully as the 'cauliflower sign' might suggest abdominal cocoon syndrome preoperatively.

\section{REFERENCES}

1. Naniwadekar RG, Kulkarni SR, Bane P, Agrarwal S, Garje A. Abdominal cocoon: an unusual presentation of small bowel obstruction. J Clin Diagn Res 2014;8(2):173-174. Frost JH, Price EE. Abdominal cocoon: idiopathic sclerosing encapsulating peritonitis. BMJ Case Rep 2015;2015:bcr2014207524.

Akbulut S. Accurate definition and management of idiopathic sclerosing encapsulating peritonitis. World J Gastroenterol 2015;21(2):675-687.

Lasheen O, ElKorety M. Abdominal cocoon or encapsulating peritoneal sclerosis: a rare cause of small bowel obstruction. Eur J Case Rep Intern Med 2020;7(12):001972.

5. Li N, Zhu W, Li Y, Gong J, Gu L, Li M, et al. Surgical treatment and perioperative management of idiopathic abdominal cocoon: single-center review of 65 cases. World J Surg 2014;38(7):1860-1867.

6. Ibrarullah M, Mishra T. Abdominal cocoon: "cauliflower sign" on CT scan. Indian J Surg 2016;78(3):243-244

7. Lim MC, Chotai NC, Giron DM. Idiopathic sclerosing encapsulating peritonitis: a rare cause of subacute intestinal obstruction. Case Rep Med 2016;2016:8206894.

8. Solak A, Solak I. Abdominal cocoon syndrome: preoperative diagnostic criteria, good clinical outcome with medical treatment and review of the literature. Turk J Gastroenterol 2012;23(6):776-779.

9. Tannoury JN, Abboud BN. Idiopathic sclerosing encapsulating peritonitis: abdominal cocoon. World J Gastroenterol 2012;18(17):1999-2004. 